\title{
AN EXPLANATION FOR THE EUV SPECTRUM OF FEIGE 24
}

\section{S. Vennes, P. Chayer, G. Fontaine, and F. Yesemael}

Département de Physique, Université de Montréal

Feige 24 is a bright DA white dwarf which has been studied extensively both from ground-based and space-borne observatories. The best determination of its fundamental atmospheric parameters are that of Holberg. Hesemael, and Basile [1986] who have used detailed model atmosphere analyses in conjunction with optical, IUE, and Voyeger data. They give log $g-7.23 \pm 0.35$ and $T_{0}\left(10^{3} K\right)-55 \pm 5$. The question of the atmospheric composition is more involved as small traces of heavy elements would not be observable in the optical spectrum of such a hot, hydrogendominated atmosphere. If it were isolated. Feige 24 would presumably only show the usual bland optical spectrum of a typical DA white dwarf, i.e. the hydrogen Balmer line series, but the presence of a $M$ dwarf companion complicates its spectrum. On the other hand, Feige 24 belongs to a handful of hot DA white dwarfs sufficiently bright that ultraviolet spectroscopy in the high resolution mode of the IUE has been possible. Following the theoretical expectation of Vouclair, Vauclair, and Greenstein (1979), it was discovered that the photosphere of Feige 24 contains small amounts of C, N, and Si (Dupree and Raymond 1982). Spectral synthesis techniques used by Wesemael, Henry, and Shipman (1984) indicate the following abundances: $\log (C / H)=-6.4 \pm 0.6, \log (N / H)=-5.3 \pm 1.0$, and $\log (\mathrm{Si} / \mathrm{H})=-6.3 \pm 0.9$. The most plausible explanation to account for these small abundances is the influence of selective radiative forces possibly coupled to a weak wind (Chayer et al. 1987).

Further interest in Feige 24 was generated by the publication of the exciting EXOSAT observations of that star (Paerels et el. 1986). Feige 24 was already known to be a strong EUV source (Margon et a/. 1976), but EXOSAT revealed for the first time an unexpected spectrum in that wavelength range. Indeed, the observed EUV spectrum of Feige 24 has, until now, defied any explanation. It consists of an essentially featureless continuum which shows a maximun around $250 \mathrm{~A}$ and which can be nominally fitted with a black body energy distribution with $T=31,000 \mathrm{~K}$, assuming an interstellar neutral hydrogen column density of $n_{H}-1.2 \times 10^{19} \mathrm{~cm} 2$ (Paerels et a/. 1986). These authors have ruled out (1) a pure hydrogen spectrum, (2) a spectrum for a hydrogen atmosphere containing uniform traces of helium, and (3) a spectrum for a hydrogen atmosphere containing the small traces of $\mathrm{C}, \mathrm{N}$, and $\mathrm{Si}$ at the levels determined by Hesemael, Henry, and Shipman (1984). Paerels et a/. (1986) concluded by pointing out to the need for more sophisticated model atmospheres including, in particular, chemical stratification. 
As a natural follow-up to our recent detailed investigation of mechanisms that could account for the purported presence of helium in the photospheres of hot DA stars (Vennes et al 1988), we have computed a grid of stratified model atmospheres consisting of a layer of pure $H$ sitting on top of a layer of pure $H_{e}$ in diffusive equilibrium (Vennes, Fontaine, and Hesemael 1988). The question that arises is whether or not such a stratification could account for the EUV spectrum of Feige 24. The basic idea here is that the hydrogen layer must be sufficiently thick that a pure hydrogen spectrum is formed in the optical region, and, yet, sufficiently thin that the EUV flux escaping from deeper layers is regulated by the opacity of helium in these regions. Our calculations show that the EUV spectrum of Feige 24 cannot be explained in terms of a $\mathrm{H} / \mathrm{He}$ stratified atmosphere.

Fig. 1 shows the count rate spectrum of Feige 24 as observed with EXOSAT (crosses). In a manner very similar to Fig. $2 a$ of Paerels et a/. (1986), we show the predictions of hydrogen model atmospheres with uniform traces of helium ( $\mathrm{He} / \mathrm{H}$ $=10^{-4}$ and $10^{-5}$, respectively). These illustrative models have $\log 9=8.0, T_{e}=50,000 \mathrm{~K}$, and a value of $n_{H}=3.5 \times 10^{18}$ is assumed. The continuum lines correspond to theoretical convolved spectra taking into account the response of the spectrometer and its effective area, and degraded by a typical FWHM resolution of 6 A. Fig. 1 shows clearly why Paerels et al. (1986) rejected hydrogen models with uniform traces of helium for Feige 24.

By comparison, Fig. 2 illustrates the predictions of layered model atmospheres having the same basic parameters as those shown in Fig. 1. Two models are shown, one with a hydrogen layer so thick $(\log \Delta M(H) M M=-14)$ that the EUV spectrum is practically the same as that of a pure $H$ model, and one with a thinner hydrogen layer $(\log \Delta M(H) M=-15)$ which allows helium to pollute the EUV photosphere and reduce considerably the flux at short welengths. Note that spectroscopy can easily distinguish between a layered and a uniform $\mathrm{H} / \mathrm{He}$ atmosphere. However, the layered atmosphere hypathesis must be rejected for Feige 24 for fundamentally the same reason as for uniform models: if helium is to be sufficiently abundant to quench the short-wavelength flux, it also leaves an obvious signature, namely an absorption edge at 228 A wich is not observed in this particular star.

This result has prompted us to consider an alternate possibility which we have alluded to in Vennes et a/. (1988). It has been recognized at the outset by the various workers in the field that the required soft $X$-ray/EUV opacity source needed to explain the observations of hot DA stars may not be totally provided by helium but also, in large part, by heavier elements. It was for reasons of simplicity that helium became the favorite absorber. In the case of Feige 24, the known presence of $\mathrm{C}, \mathrm{N}$, and Si has remained suggestive oven though Paerels et \&. (1986) concluded that models with traces of these elements could not explain the observations. This is because the IUE window can only reveal a relatively small number a metallic resonance lines, and the detection of only $\mathrm{C}, \mathrm{N}$, and $\mathrm{Si}$ in the /UE spectrum of Feige 24 cannot be interpreted as a lack of other elements. Quite the contrary, if 
small abundances of $C, N$, and $S i$ are (presumably) supported by radiative levitotion, we expect that a host of other elements can be supported too. Thus, we envision a situation in which many different metals with low abundances ore present in the atmosphere of Feige 24. These metals would lead to a large number of small absorption edges which are smoothed over becouse of the finite resolution of $\overline{E \times O}$ $S A T$ ( 6 A), and whose cumulative effect is to quench the short-wavelength flux.

As an illustrative example, we have computed the synthetic EUV spectrum of a model of Feige 24 with $\log g-723$ and $T_{e}=55,000 \mathrm{~K}$. The model is H-dominated with small uniform traces of 10 elements: $\mathrm{He}, \mathrm{C}, \mathrm{N}, \mathrm{O}, \mathrm{Ne}, \mathrm{Na}, \mathrm{Si}, \mathrm{S}, \mathrm{Ar}$, and $\mathrm{Ca}$. These particular elements were chosen for the presence of photoionization edges in the 190-350 A EXOSAT range and for the absence of resonance lines (except for $C$, $N$, and $\mathrm{Si}$ ) in the 1200-3000 A IUE range at the effective temperature of interest. It is important to note that the abundances that were chosen are entirely consistent with the abundances which we have computed for each element from radiative support theory. Some adjustments of individual abundances have been made, but this does not change the central result of our experiment.

Fig. 3 contrasts the predicted spectrum (continuous line) with the observed EUV count rate spectrum of Feige 24. Our synthetic spectrum is normalized to the visual magnitude of the object $(V=12.56)$, so that the result presented in Fig. 3 is an absolute fit, not a simple fit to the shape of the EUV spectrum. The agreement is gratifying. Of course, we cannot pretend that there exists a unique fit; our choice of absorbers is somewhat arbitrary and other possibilities exist. He have left out a potentially great number of absorbers, and a detailed comparison at the level of emall residual features in the theoretical curve cannot realistically be made. In fact, the prospect for such a comparison remains unlikely until we understand in details what governs the relative and absolute abundances of trace species in the atmospheres of hot white dwarfs. He know that the predictions of simple radiative support theory -which assumes a perfect equilibrium between gravitational settling and radiative levitation- are not consistent with the observations. It is thought that weak winds play havoc with the abundances of metals supported by radiation in hot white dwarfs (Chayer, Fontaine, Hesemael 1988). Until we have a decent theory of these phenomena, we must remain satisfied with the qualitative picture described here. He strongly believe, however, that the basic idea is correct: the EUV spectrum of Feige 24 can simply be explained by the cumulative effects of a host of trace absorbers in a hydrogen atmosphere.

If this idea is indeed correct, then it raises a number of interesting questions concerning the interpretation of EUV/soft $X$-ray data in terms of layered atmospheres. In particular, is Feige 24 an exception or a typical DA white dwarf $?$ A qualitative comparison between the DA stars observed in the EUV range as discussed by Vennes et $a /$. (1988) and the sample of DA objects in the high resolution /UE mode by Bruhweiler and Kondo (Bruhweller 1985) is of interest here. He find, from the limited information available, that seven objects out of seven which require a 
EUV opacity source also show some metallic features in their /LE spectra. Are these all similar to Feige 24 in the sense that their EUV fluxes are regulated by a cumulative metallic opacity? In that case, there would no need to invoke thin hydrogen layers for those stars. We find this possibility rather suggestive, but we cannot be certain because, except for Feige 24, we do not know the abundances of the elements detected with the IUE. This is underscored by the puzzling fact that we also find three objects in the EUV sample which do not require a EUV opacity source (i.e. they emit like pure hydrogen models), and, yet, also show metallic features in their IUE spectra. At the very least, this suggests that the abundances of the metals detected must be very small in order not to quench significantly the EUV flux. Thus, the presence of metals in the IUE spectra of a DA star does not necessarily eliminate the need for $\mathrm{H} / \mathrm{He}$ layered atmospheres.

In that context, it should be remembered that the case for layered atmospheres is very strong for the DAO stars which are hot progenitors of ordinary DA stars. Vennes et $a /$. (1988) find that this model is the only viable one for that type of objects. As evolution proceeds, DAO stars cool to become ordinary DA stars and, therefore, must retain their layered configurations. The observations of the DAO object PG $1210+533$ by Holberg et a/. (1988) reinforce strongly the conclusion of Vennes et a/. (1988). Thus, it may very well be the case that the effects of both H/He stratification and metallic absorption regulate the EUV flux of hot DA white dwarfs. The opacity could be dominated in some stars (such as Feige 24) by metallic absorption, in other stars by $\mathrm{H} / \mathrm{He}$ stratification (in cases where a wind has expelled the heavy elements from the star for example), and in other cases by both mechanisms. Before the question is definitely settled, we will have to await the results of future experiments which should provide the definitive test. The available Elnstein and EXOSAT photometric observations can currently be understood either within the framework of $\mathrm{H} / \mathrm{He}$ layered atmospheres or within the framework of metallic absorbers. The answer lies with EUV spectroscopy, since uniform models, stratified models, and models with metals have quite different spectral signatures.

This research has been supported in part by the NSERC Canada, by the fund FCAR (Québec), and a E.WR. Steacie Memorial Fellowship to one of us (GF).

Bruhweiler, F.C. 1985, Bull. Amer. Astran. Soc., 17, 559.

Chayer, P., Fontaine, G., Wesemael, F. and Michaud, G. 1987, in IAU Colloquium No. 95, The Second Conference on Faint Blue Stars, A. G. D. Philip, D. S. Hayes and J. Liebert, eds., L. Davis Press, Schenectady, p. 653 .

Chayer, P., Fontaine, G., and Wesemael, F. 1988, these proceedings. Dupree, A.K., and Raymond, J.C. 1982, Astraphys. J. Letters, 263, L63. Holberg, JB., Hesemael, F., and Basile, J. 1986, Astrophys. J., 306, 629. Holberg, JB., Sion, EM., Liebert, J., and Vauclair, G. 1987 Bu/l. Amer. Astron. Soc., 19, 1041.

Margon, B., Lampton, M., Bowyer, S., Stern, R., and Paresce, J. 1986 Astrophys. J. 
Letters, 210, L79.

Poerels, FB.S., Bleeker, JAM., Brinkman. A.S., and Heise, J. 1986 Astrophys. J. Letters, 30s, L33.

Vauclair. G., Vauclair, S.. and Greenstein, JL. 1979, Astron. Astraphys., $80,79$.

Vennes, S., Pelletier, C., Fontaine, G., and Hesemael, F. 1988, Astrophys. J., in press.

Vennes, S., Fontaine, G., and Hesemael, F. 1988, these proceedings.

Hesemael, F., Henry, RB.., and Shipman, HL., 1984, Astrophys. J., 287, 868.

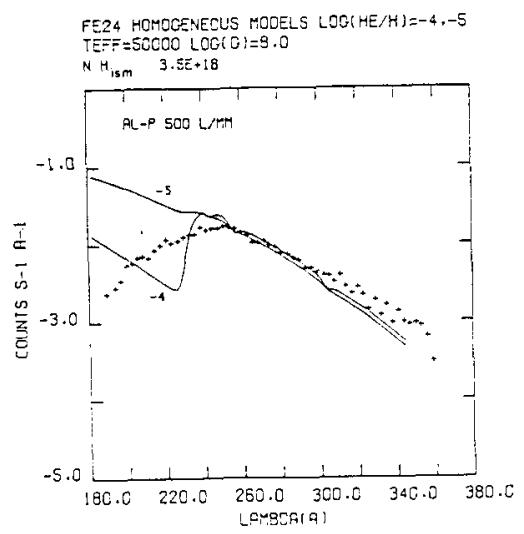

Figure 1. Synthetic spectra of
homogeneously mixed H-He atmospheres
convalved with the spectrometer response and
compored with the measured spectrum (crosses).
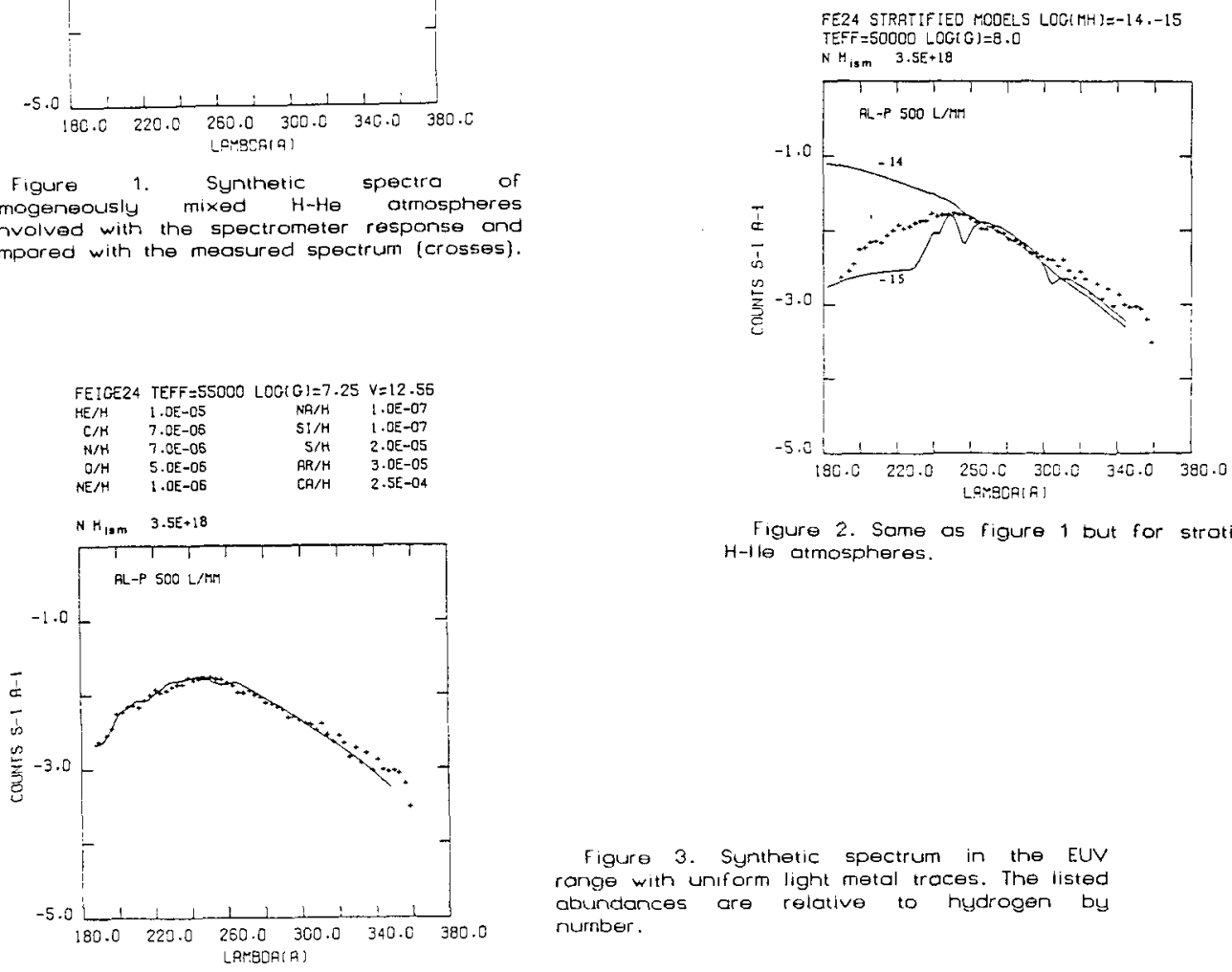

Figure 2. Same as figure 1 but for strotified $H-1$ le atmospheres.

Figure 3. Symthetic spectrum in the EUV ronge with uniform light metal traces. The listed abundances are relative to hydrogen by number. 